\title{
Review Article \\ The Clinical Efficacy of Yindanxinnaotong Soft Capsule in the Treatment of Stroke and Angina Pectoris: A Meta-Analysis
}

\author{
Long Cheng, ${ }^{1}$ Yue Liu, ${ }^{2}$ and Xiao-bo Sun ${ }^{1}$ \\ ${ }^{1}$ Key Laboratory of Bioactive Substances and Resources Utilization of Chinese Herbal Medicine, Ministry of Education, \\ Institute of Medicinal Plant Development, Chinese Academy of Medical Sciences and Peking Union Medical College, \\ Beijing 100193, China \\ ${ }^{2}$ Xiyuan Hospital, China Academy of Chinese Medical Sciences, Beijing 100053, China
}

Correspondence should be addressed to Xiao-bo Sun; sun-xiaobo@163.com

Received 2 November 2016; Revised 2 March 2017; Accepted 20 March 2017; Published 30 April 2017

Academic Editor: Gioacchino Calapai

Copyright (C) 2017 Long Cheng et al. This is an open access article distributed under the Creative Commons Attribution License, which permits unrestricted use, distribution, and reproduction in any medium, provided the original work is properly cited.

Objective. To systematically evaluate the clinical efficacy of Yindanxinnaotong (YD) soft capsule in adult patients with cardiovascular diseases (stroke and angina pectoris). Methods. We electronically searched databases including Medline, PubMed, Chinese National Knowledge Infrastructure (CNKI), Cqvip Database (VIP), and Wanfang Database for published articles of randomized controlled trials (RCTs) of YD capsule in treating stroke and angina pectoris. The meta-analysis was performed using RevMan 5.3 software. Results. 49 RCTs involving 6195 subjects with cardiovascular diseases (angina pectoris and stroke) were included. Compared with western conventional medicine (WCM) and/or other Chinese medicines, YD plus WCM therapeutic regimen could significantly improve the efficacy rate $(\mathrm{RR}=1.21,95 \% \mathrm{CI}(1.17,1.25), P<0.00001$ for angina pectoris, $\mathrm{RR}=1.24,95 \% \mathrm{CI}(1.18$, 1.31), $P<0.00001$ for stroke), showing the clinical value. In addition, the therapeutic efficiency of WCM plus YD capsule regimen is better than that of WCM alone in improving CRP $(\mathrm{MD}=-2.07,95 \% \mathrm{CI}(-3.97,-0.17), P=0.03<0.05)$ and TG $(\mathrm{MD}=-0.37$, $95 \%$ CI $(-0.52,-0.23), P<0.0001)$. Conclusion. YD is effective in the treatment of cardiovascular diseases (angina pectoris and stroke) in adults, and WCM plus YD therapeutic regimen can significantly improve the effective rate in the clinic.

\section{Introduction}

Stroke and coronary heart disease (CHD) are the leading causes of morbidity, mortality, and health care expenditure in adults especially the elderly. Both diseases share risk factors and pathogenic processes, that is, atherosclerosis and thrombosis. Even if the most advanced, comprehensive treatment is applied, still more than $50 \%$ of the survivors from cerebrovascular accidents cannot live completely and take care of themselves [1,2]. Stroke and angina pectoris have the characteristics of high incidence, high mortality, high morbidity, high recurrence rate, and complications. At present, China has more than 270 million patients with stroke and angina pectoris. Nearly 3 million patients with stroke and angina pectoris died in China every year, accounting for 51\% of China's annual total death [3].

Yindanxinnaotong (YD) capsule is a Chinese patent drug processed by modern advanced pharmaceutical technology with improved formulations, which refined the characteristics of traditional Miao ethnic medicine. The formulations of YD include ginkgo leaves, salvia miltiorrhiza, herba erigeromtis, Gynostemma pentaphyllum, hawthorn, allium sativum, Panax notoginseng, and borneol [4]. YD has the function of promoting blood circulation, removing blood stasis, and relieving pain [3]. YD has the indications of Qi stagnation and blood stasis caused by obstruction of Qi in the chest; and treatment includes chest pain, chest tightness, shortness of breath, and heart palpitations and angina pectoris of coronary heart disease, hyperlipidemia, cerebral arteriosclerosis, stroke, stroke sequelae, and so forth. Modern pharmacological studies have shown that YD can expand coronary artery, increase the coronary blood flow, smooth vascular muscle, reduce myocardial consumption oxygen, prevent and treat myocardial ischemia, reduce platelet aggregation and blood lipids, and protect from the ischemia reperfusion injury $[5,6]$. 
In China, Chinese medicines play an irreplaceable role in the prevention and treatment of cardiovascular diseases, and more and more doctors tend to apply the combined regimen (western conventional medicine (WCM) plus Chinese medicine), to improve cardiovascular disease treatment outcome [7-10]. However, the safety and effectiveness of Traditional Chinese Medicine (TCM) in clinical applications are not unified and need more scientific and standardized assessment data to provide reliable evidence for its clinical practice $[11,12]$. Given present situation, the research aims to evaluate the appropriate use of YD capsule in treatment of stroke and angina pectoris through meta-analysis and literature research methods and promote the rational use of YD. Researches have confirmed that the high sensitive C-reactive protein (hs-CRP) and triglyceride (TG) are closely related to the development of atherosclerosis. In this study, we also comprehensively evaluated the effect of Yindanxinnaotong capsule on the above indexes.

\section{Material and Methods}

2.1. Inclusion and Exclusion Criteria. Studies of randomized controlled trials were included, the published language is not limited, regardless of whether the study was blinded in grouping. The specific inclusion criteria were the following.

(1) Subjects were included and diagnosed as stroke or angina pectoris.

(2) The treatment group received western conventional medicine (WCM, Table 1) plus YD capsule treatment; the control group received WCM alone.

(3) All included research literature must be able to extract one of the following results: the primary outcome was angina pectoris symptoms, clinical efficacy, or nerve function defect score; the secondary outcome measures included electrocardiogram, angina attack frequency and duration, blood rheology, and lipids characters.

The exclusion criteria were the following.

(1) The included literatures were nonrandomized, controlled trials.

(2) The studies being animal experiments or clinical pharmacokinetic studies, or the study enrolling healthy subjects.

(3) The research data has obviously large deviation.

(4) The design scheme of the research is not clear, or the data is not complete.

2.2. Interventions. The interventions regimen was WCM plus YD treatment or WCM treatment alone. (1) WCM treatment of coronary heart disease and angina pectoris includes nitrates, aspirin, and amlodipine; (2) WCM treatment for stroke includes nimodipine, flunarizine, and aspirin; (3) angina pectoris routine treatment includes nitrous esters, aspirin, amlodipine, and isosorbide dinitrate; (4) the treatment dose of YD capsule was referenced according to drug directions; the courses ranged from 2 to 12 weeks.
The final outcome/expected outcome criteria should clearly include at least one of the following outcomes: (1) at the end of treatment or follow-up period, clinical symptoms ease, ECG efficacy or other clinical symptoms of improvement, or the total clinical efficiency rate was expressed; (2) the therapeutic effect was compared as blood lipids (total cholesterol, glycerin trilaurate, low density lipoprotein cholesterol, and high density lipoprotein cholesterol) changes at the end of treatment or at follow-up end; (3) score of blood rheology index changes exist (plasma specific viscosity, fibrinogen, and C-reactive protein); (4) the change of Barthel index score, neurological deficit score, and life ability score exist in stroke at the end of treatment or follow-up.

Literature Source. The literatures were collected through computer retrieval system, the database including PubMed, Medline, China Biology Medicine disc (CBMDisc), Chinese Medical Academic Conference papers database (CMAC), China National Knowledge Infrastructure (CNKI), Chinese science and technology journal full-text database (VIP), Web of Science, and Wanfang Database from 2007 to 2016; the retrieval deadline is June 2016. And all literatures that met the inclusion criteria of references and are relevant to YD capsule in the cardiovascular prognosis were searched one by one for data missing.

Retrieval Method. We screened above retrieval database with the key words of "YD capsule", "Yindanxinnaotong capsule", and YDXNT capsule; "angina pectoris" and "stroke". The language was not restricted; limited conditions include "human" and "random of test". We also use Google Scholar, Baidu scholar, and other search engines and manual retrieval as a supplement to trace included references. Retrieved literatures are imported into duplicate check software in order to remove duplication and manage document.

Literature Selection and Data Extraction. There were 2 reviewers independently screening and cross-checking these literatures according to predefined inclusion criteria. Disagreement was addressed to a third researcher. The lack of information was solved through contacting the original author. Through screening the title, abstract, and text, reviewers determined whether the final data could be included. The mainly included contents were basic information of the study, including research title, first author, published journal, and time; the key elements of study design and quality evaluation; the basic situation of experimental group and the control group, including the number of cases included and age; the effectiveness, safety, and so on.

Bias Risk Assessment. The bias risk assessment was performed by 2 reviewers in accordance with the Cochrane collaboration group for randomized controlled trials by tools [13, 14]. The evaluated contents included (1) random allocation method; (2) allocation of groups; (3) the blind method applied in research subjects and researchers; (4) the results of data integrity: including baseline measurements before the intervention and effect parameters after intervention, drop out/exit (whether the dropout rate is less than 10\%), 


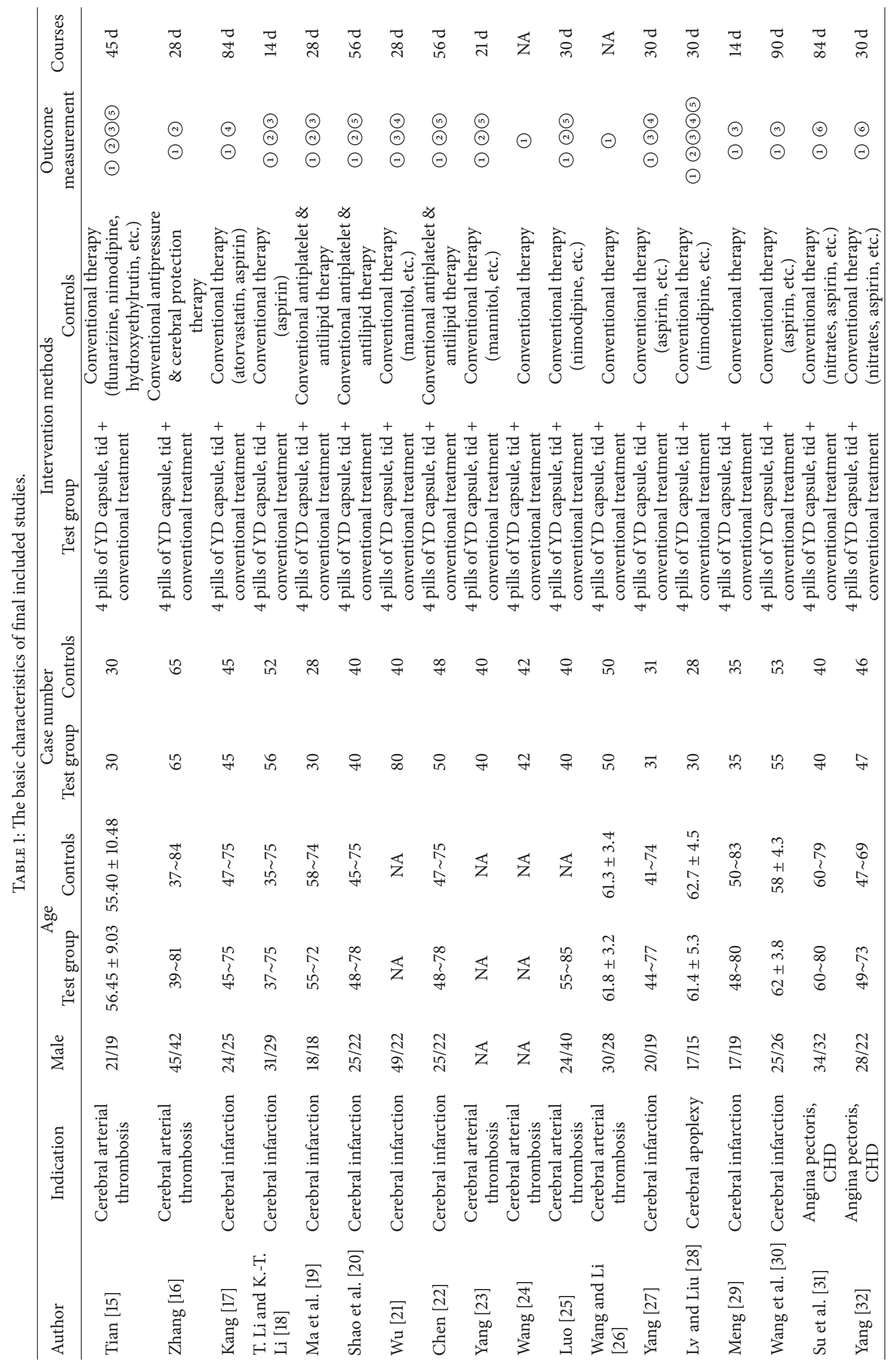




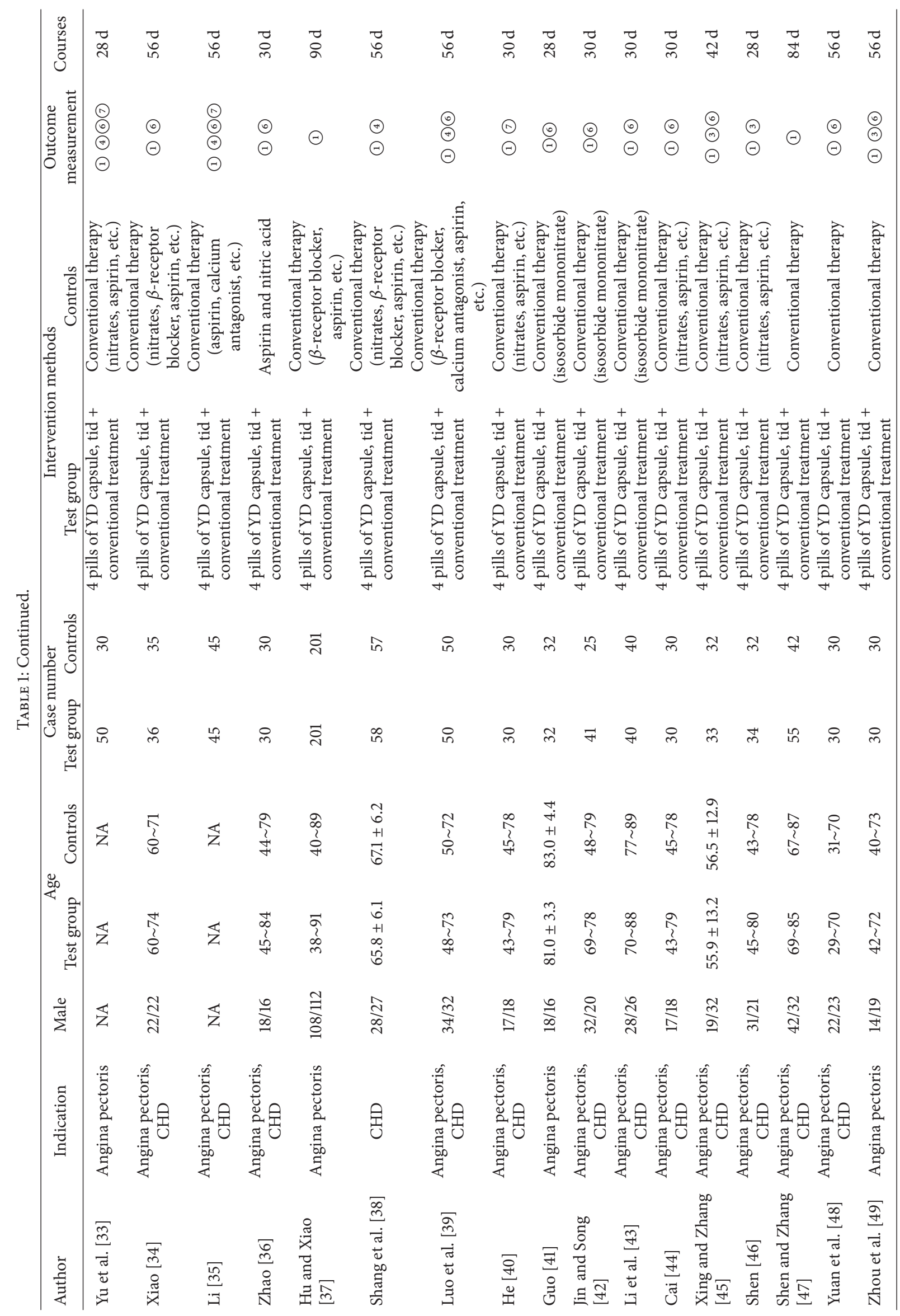




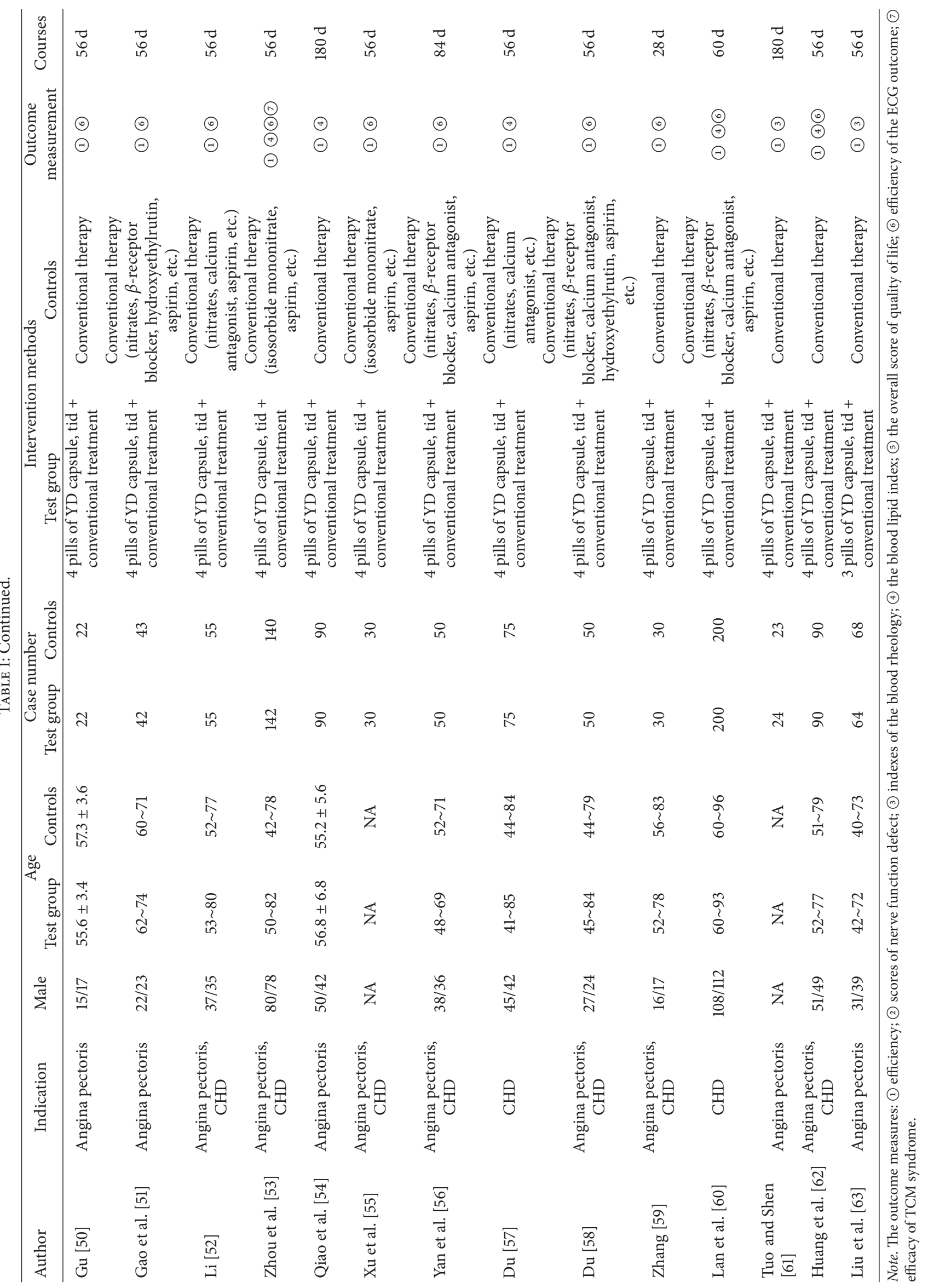




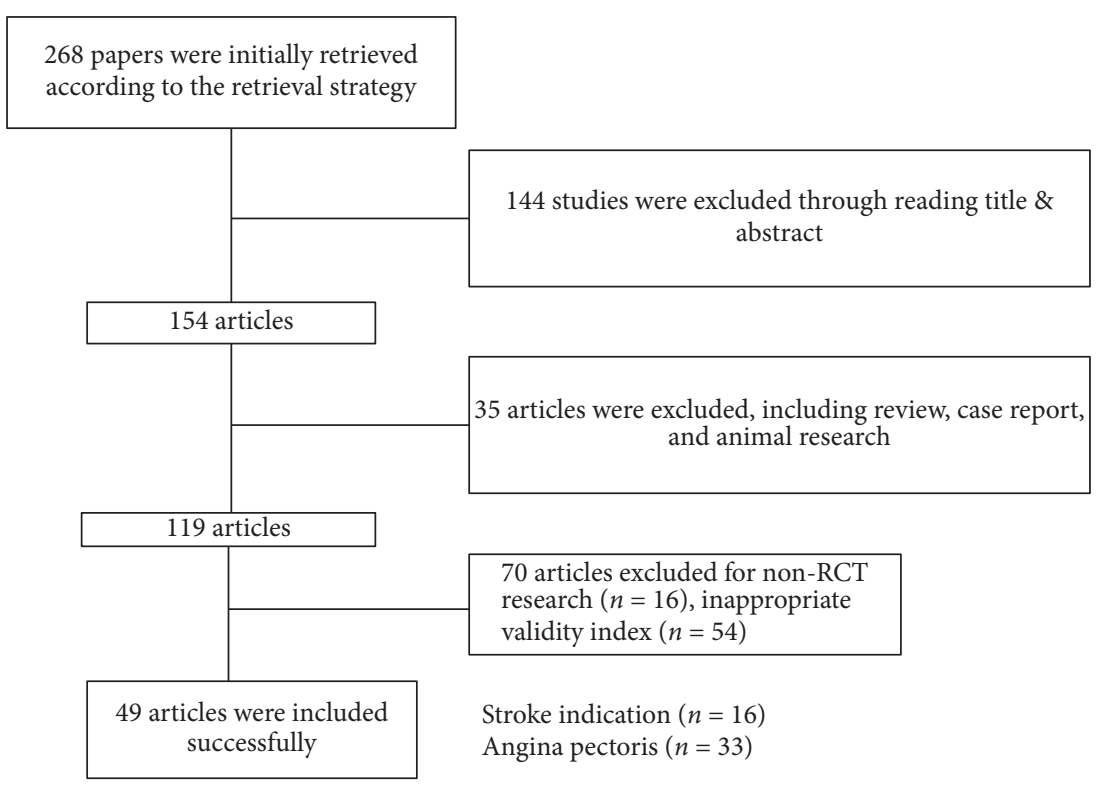

FIGURE 1: The literature screening result.

and whether the loss reason was explained whether drop out/existing patients' data was analyzed by the intent to treat (ITT) analysis; (5) the selective reporting results: for the negative result, whether the security issues (death and other adverse events) were reported; (6) other sources of bias including early stop of test and baseline imbalance. "Yes" in point (6) means the bias was low, "no" means high bias, and "unclear" means the lack of relevant information or bias uncertainty.

Data Analysis. The RevMan 5.3 software was used for statistical analysis. The binary classification data were expressed as odds ratio (OR) and 95\% CI. The continuous data were expressed as mean difference (MD) and 95\% CI. Chi square test (alpha $=0.1$ ) was used for heterogeneity analysis and the heterogeneity of size was quantitatively assessed combined with $I^{2}$. If $P>0.05, I^{2} \leq 50 \%$, fixed effects model was used; if there is heterogeneity, the sources of heterogeneity should be examined and removed; if the heterogeneity still exists, and the study has clinical homogeneity, meta-analysis was performed using random effect model. If the heterogeneity could not be combined, the descriptive analysis was adopted.

\section{Result}

According to retrieval strategy, a total of 268 related literatures were retrieved, through reading the title and abstract, and 114 articles were excluded for not meeting the inclusion criteria and involving animal study. 35 articles containing duplicated research, nonrandomized control study, interventions, and end index which does not meet inclusion criteria were removed; then, the other 5 literatures with clear deviation of outcome indicators were removed, and 119 articles were included for further review. The final included RCTs were 49 (Figure 1).
3.1. The Characteristics of Included Study and the Risk Assessment of Bias. The 49 RCT studies [15-63] included 6195 patients with stroke and angina pectoris. The minimum sample size of each group was 22 , the maximum sample size was 201. The basic characteristics of the included studies are shown in Table 1, and the bias risk assessment results are shown in Table 2.

3.2. Stroke Curative Effect Analysis. A total of 1384 patients in 16 random control trial (RCT) [15-30] were included. The fixed effect model in meta-analysis showed that the therapeutic effect of WCM plus YD capsule (in 719 patients) regimen is better than WCM alone regimen treatment (in 665 patients $)(\mathrm{RR}=1.24,95 \% \mathrm{CI}: 1.18,1.31 ; P<0.00001)$ (Figure 2). Funnel plot (Figure 3) showed that the literature is symmetrical, with Eggers test of $P>0.05$. There is no publication bias. Results showed that YD capsule combined with conventional treatment can control and improve the clinical symptoms of stroke, indicating obvious clinical value.

3.3. Curative Effect of YD on Angina Pectoris. 3811 patients in 33 RCTs [31-63] were included: 1932 received WCM plus YD capsule and 1879 received WCM. The fixed effect model in meta-analysis showed that the therapeutic efficiency of WCM plus YD capsule regimen is better than that of WCM alone regimen treatment $(\mathrm{RR}=1.21,95 \% \mathrm{CI}: 1.17,1.25 ; P<$ 0.00001) (Figure 4). The funnel plot (Figure 5) showed that the literatures were distributed centrally and symmetrically. The Eggers test result was $P>0.05$; there is no publication bias.

3.4. Effect of YD on CRP and TG. The fixed effect model in meta-analysis showed that the therapeutic efficiency of WCM plus YD capsule regimen is better than that of WCM alone in improving CRP $[17,30,37,52]: \mathrm{MD}=-2.07,95 \% \mathrm{CI}$ 


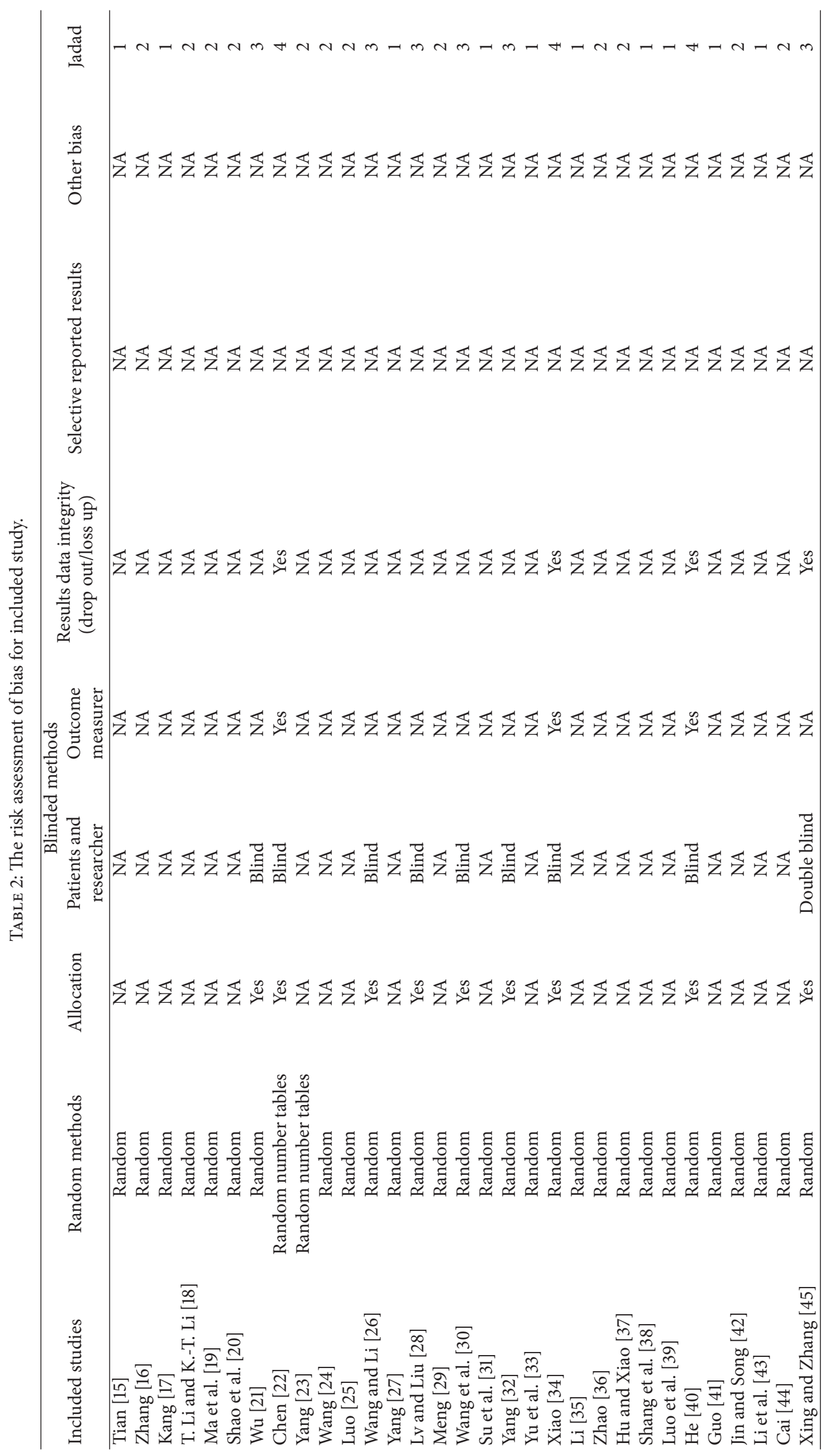




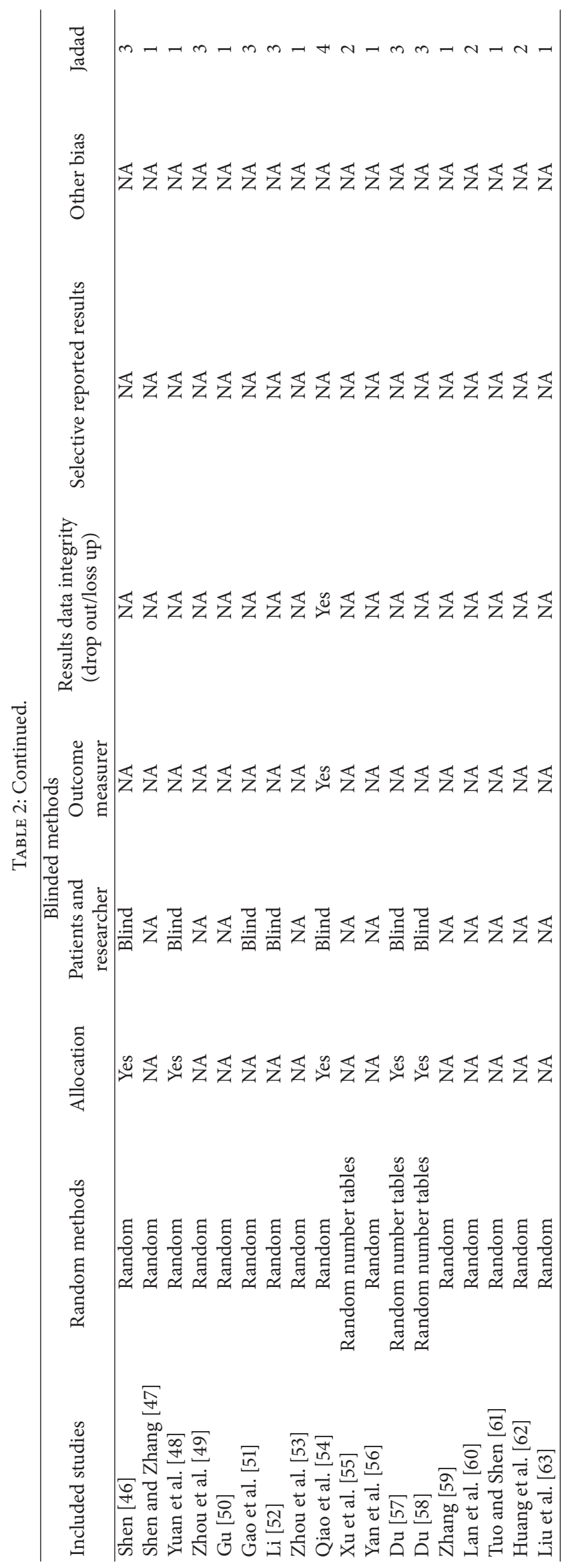




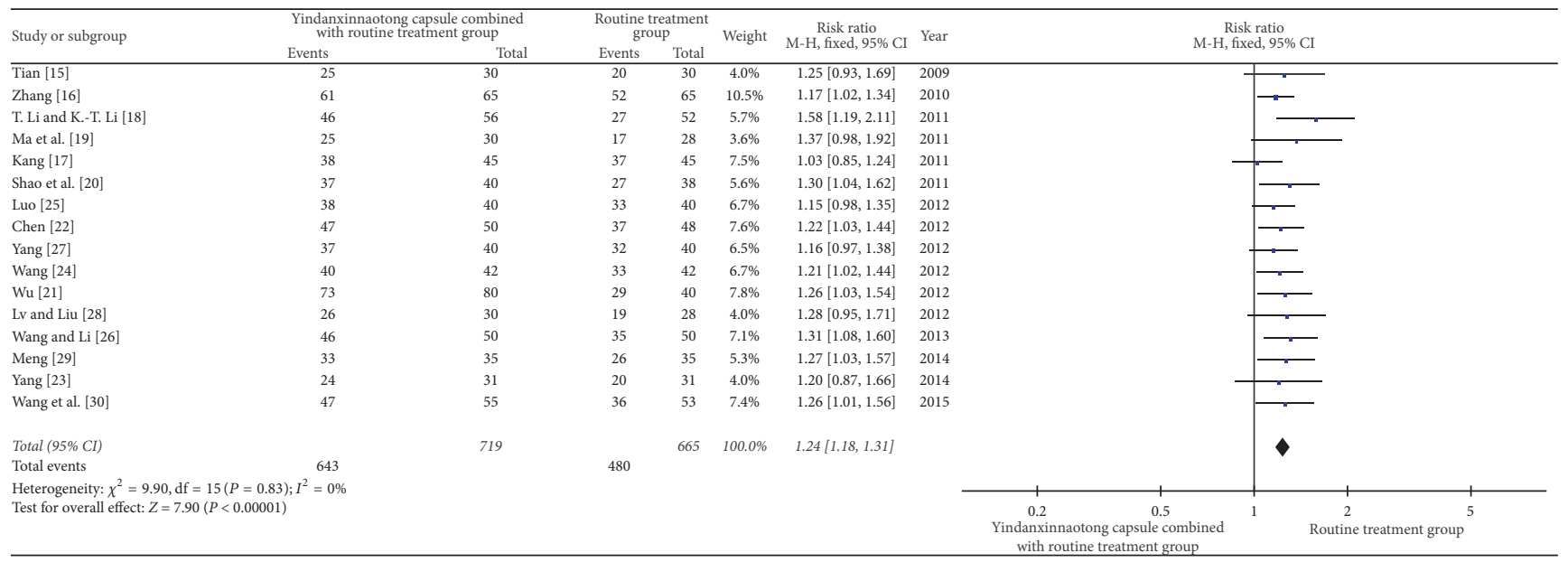

FIGURE 2: Meta-analysis of therapeutic effect of YD on stroke.

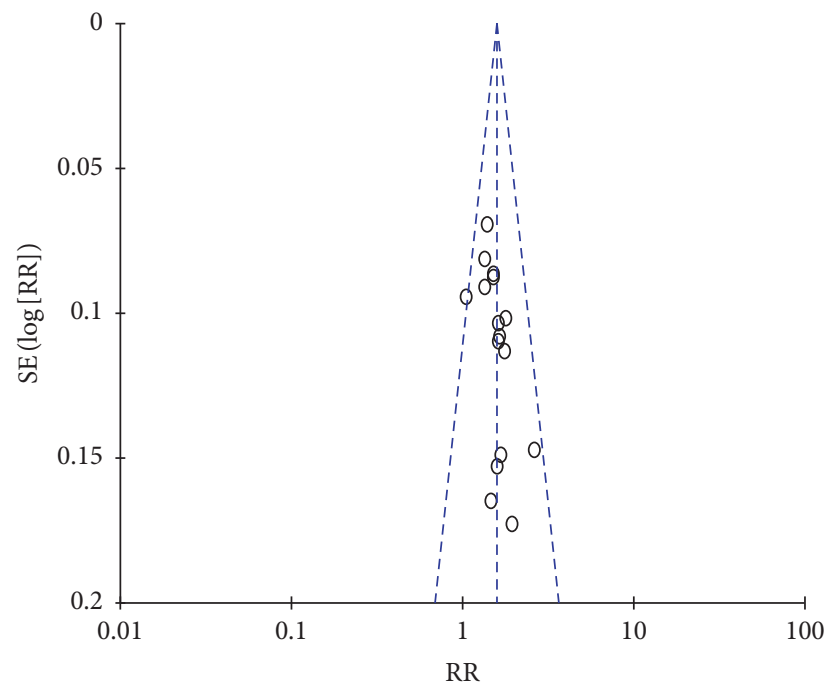

FIgURE 3: Funnel plot of the therapeutic effect of YD on stroke.

(-3.97, -0.1$), P=0.03$, Figure 6 and TG $[17,18,21,27,35,47$, $54,57,60]: \mathrm{MD}=-0.37,95 \% \mathrm{CI}(-0.52,-0.23), P<0.0001$, Figure 7.

In addition, analysis results also demonstrated that YD could regulate coagulation condition and fibrinolytic system in patients with coronary heart disease and improve myocardial ischemia, angina symptoms, and heart function. Administration of YD reduced restenosis after interventional treatment. YD can not only treat coronary heart disease, but also regulate blood lipids, which are important risk factor for coronary heart disease. Administration of YD could control the long-term development of the cardiovascular disease and prevent the cardiovascular events from happening again; YD has important significance for patients with cardiovascular disease.

\section{Discussions}

Prior to the development of modern pharmaceutical drugs, doctors of Traditional Chinese Medicine (TCM) treated angina and other symptoms of coronary artery disease through the use of acupuncture, herbal remedies, qigong, and tai chi. Today, Chinese Traditional Medicine therapies are proving to still be useful for cardiovascular disorders. Although patients may be diagnosed with different diseases according to western medicine, if the cause of the disease is the same according to TCM diagnosis and differentiation, their treatment principle will be the same.

Chinese medicine believes that cardiovascular disease is categorized to "heartache, palpitations, chest pain" in Traditional Chinese Medicine theory; and the cerebrovascular diseases could be collectively referred to as "stroke"; the weakness of blood circulation that leads to blood stasis is the important reason for the formation of cardiovascular disease. "Deficiency" is the fundamental reason of the 2 diseases and "stasis" is the direct reason. The key treatment in cardiovascular disease is to invigorate Qi, promote blood circulation, and remove blood stasis. The western medicine theory categorized lesions occurring in the cardiac artery as cardiovascular disease, like coronary heart disease, angina pectoris, myocardial infarction, and so on. Cerebral stroke, also known as the "stroke," is a group diseases of brain vessel injury due to brain vessel rupture or vascular flow obstruction. According to the change of clinical manifestations, stroke can be divided into two categories: hemorrhagic and ischemic stroke, which include commonly occurring cerebral embolism and cerebral hemorrhage in clinic [64-66]. The main pathology of the 2 diseases is atherosclerosis; the major complication of atherosclerosis is thrombosis, which could cause local arterial/distal embolization and lead to various vascular diseases $[67,68]$. The standardized treatment in western medicine is mainly to deal with cardiovascular events, including expanding coronary artery, controlling blood pressure, reducing blood lipid and blood viscosity, and anticoagulation and antiplatelet aggregation treatment.

In this paper, RCT literatures on the efficacy of YD and commonly used WCM or Chinese medicine in clinical treatment of stroke and angina pectoris have been systematically evaluated and analyzed. Results indicate that YD capsule 


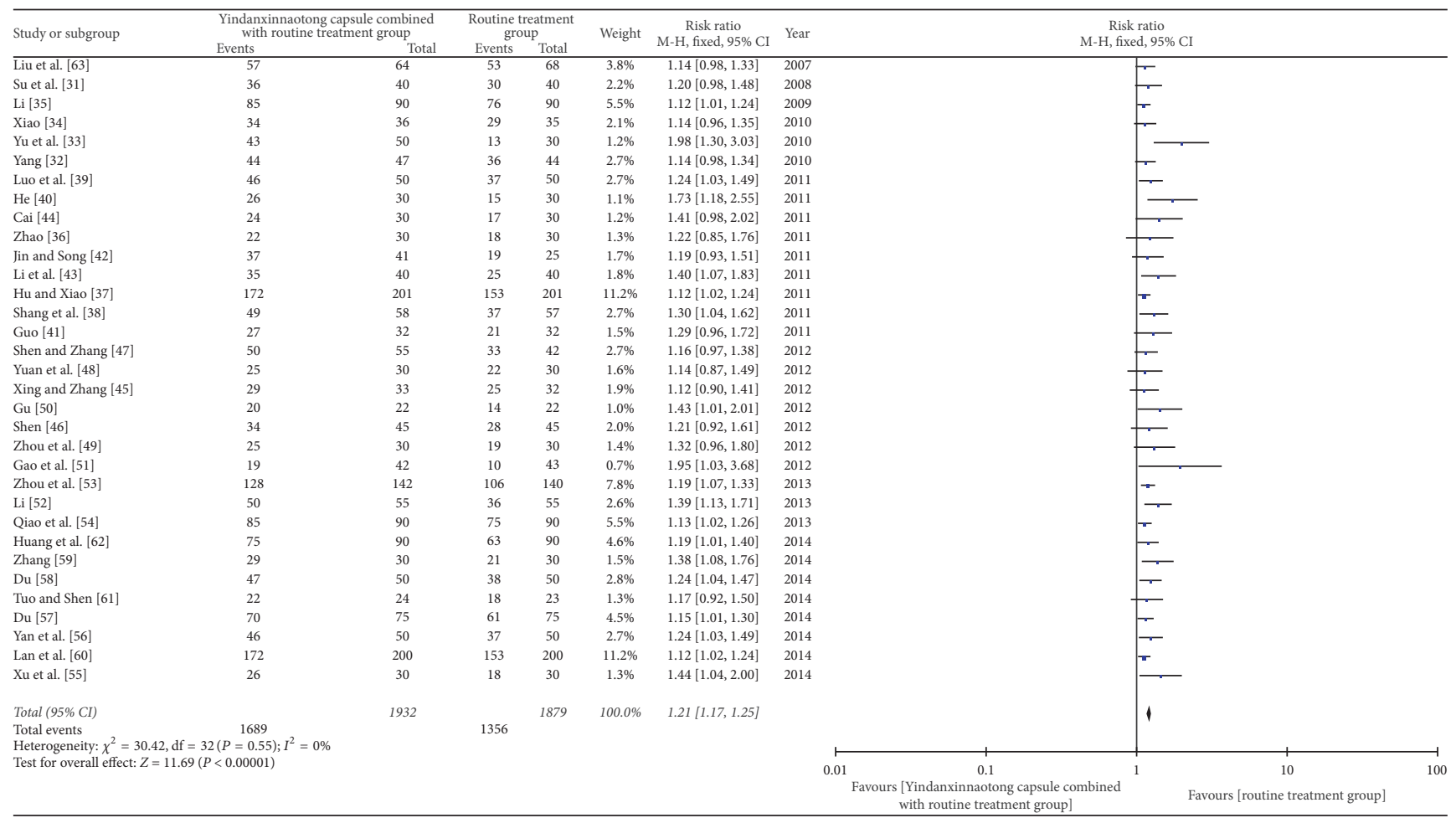

FIGURE 4: Meta-analysis of therapeutic effect of YD on angina pectoris.

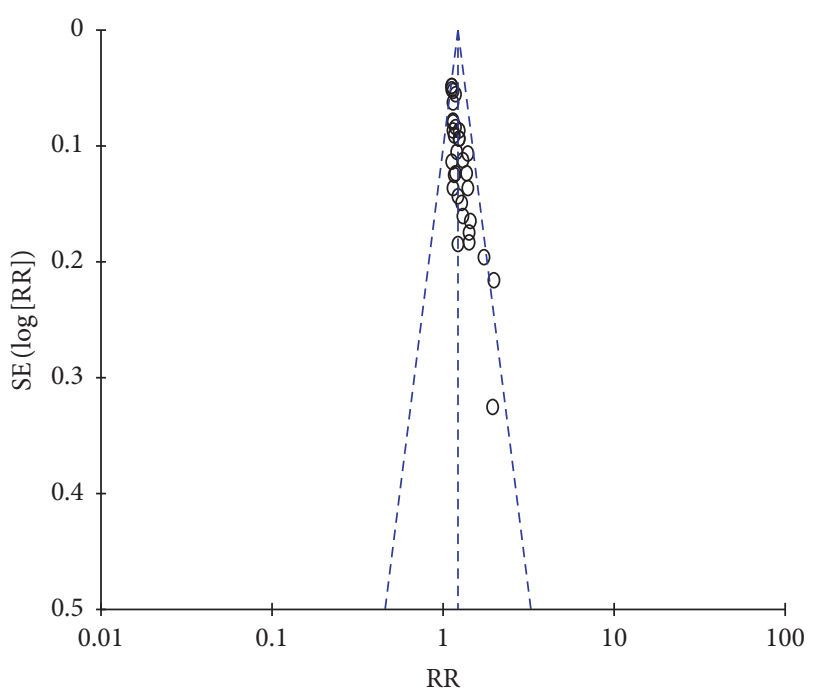

FIGURE 5: Funnel plot of the therapeutic effect of YD on angina pectoris.

combined with routine treatment has significantly higher efficiency than conventional treatment in improving clinical symptoms of patients with stroke or angina pectoris and improves the cardiovascular related laboratory index, such as
ECG, decreasing blood lipid. This might be because the YD contains multibioactive substances [69, 70]; Ginkgolides can scavenge oxygen free radicals and improve microcirculation and myocardial hypoxia [67]; Gynostemma has antiplatelet and inhibits thrombosis and blood lipid lowering effect [71]. So YD can improve the blood flow, increase cardiovascular support, repair vascular elasticity, reduce blood viscosity, keep blood flowing, and regulate blood pressure auxiliary. YD could be comprehensively used in different stages of cardiovascular disease. It is especially suited for patients with stroke and angina pectoris simultaneously; it could improve the associated symptoms caused by cardiovascular disease and regulate the blood lipid conditioning and prevent the occurrence of cardiovascular event.

In summary, for patients with stroke and angina pectoris, YD capsule combined with routine medication has certain advantages in improving clinical symptoms and laboratory indexes. Due to lack of long-term follow-up data, long-term efficacy and safety of YD can not be assessed. Therefore, the confirmative conclusions are not allowed. High-quality, large sample, multicenter randomized clinical trials still need to be done in the future.

\section{Conflicts of Interest}

The authors declare that they have no conflicts of interest regarding the publication of this article. 


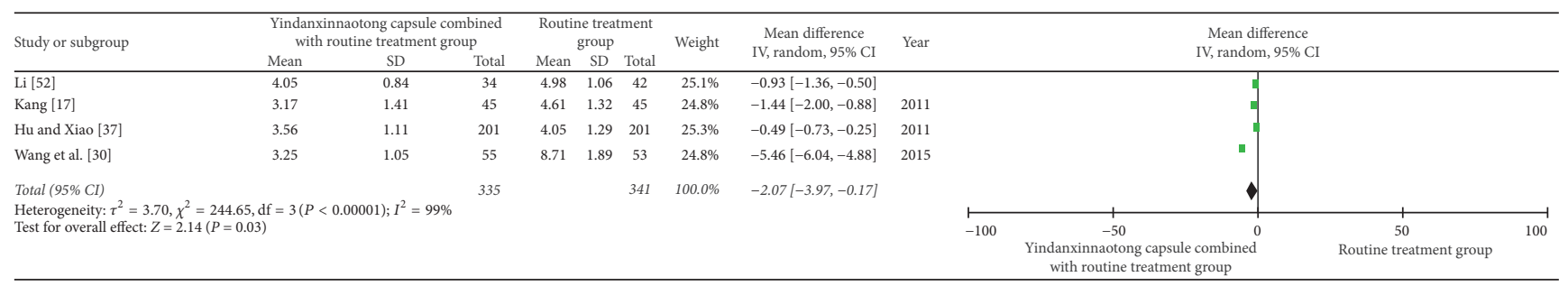

FIGURE 6: Meta-analysis of therapeutic effect of YD on CRP.

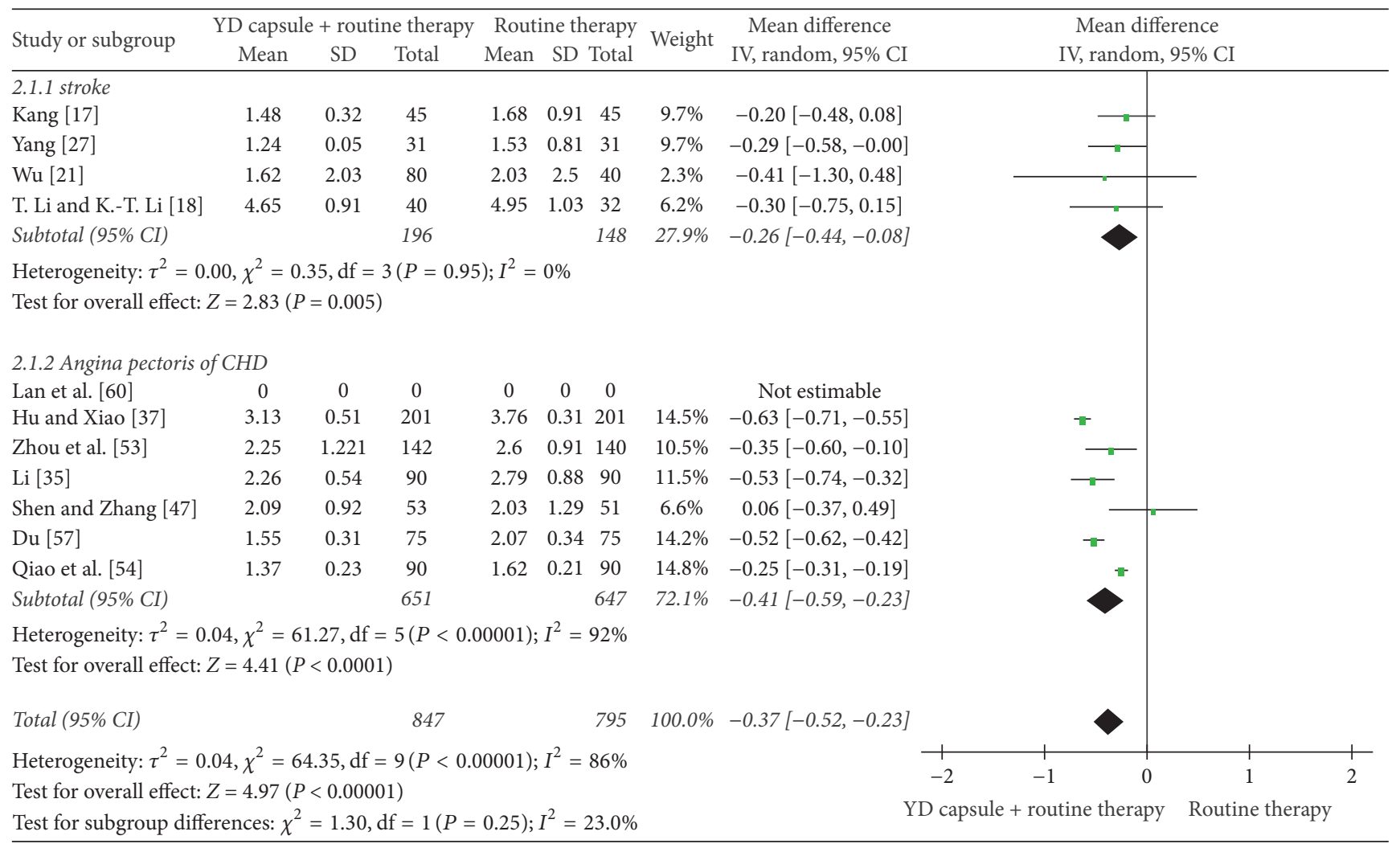

FIgURE 7: Meta-analysis of therapeutic effect of YD on TG.

\section{Authors' Contributions}

L. Cheng and X. Sun conceived and designed the protocol. Y. Liu performed the experiments. L. Cheng and Y. Liu wrote the paper. All authors reviewed and approved the submitted version of the manuscript. Long Cheng and Yue Liu contributed equally to this work.

\section{Acknowledgments}

The authors thank Dr. Li Yong for providing professional English language editing of this paper. This work has been funded by China Ministry of Science and Technology (the National Science and Technology Projects nos. 2012ZX09201201-003, 2013BAI11B01) and China Postdoctoral Science Foundation (no. 2014M552546XB).

\section{References}

[1] H. A. DeVon, K. Vuckovic, C. J. Ryan et al., "Systematic review of symptom clusters in cardiovascular disease," European Journal of Cardiovascular Nursing, vol. 16, no. 1, pp. 6-17, 2017.

[2] V. Y. Guo, B. Cao, X. Wu, J. J. W. Lee, and B. C.-Y. Zee, "Prospective association between diabetic retinopathy and cardiovascular disease-a systematic review and meta-analysis of cohort studies," Journal of Stroke and Cerebrovascular Diseases, vol. 25, no. 7, pp. 1688-1695, 2016.

[3] P. Zhang, G. Dong, B. Sun et al., "Long-term exposure to ambient air pollution and mortality due to cardiovascular disease and cerebrovascular disease in Shenyang, China," PLoS ONE, vol. 6, no. 6, Article ID e20827, 2011.

[4] L. Cheng, G.-F. Pan, X.-D. Zhang et al., "Yindanxinnaotong, a Chinese compound medicine, synergistically attenuates 
atherosclerosis progress," Scientific Reports, vol. 5, Article ID 12333, 2015.

[5] D. Li, "Effect of Yindanxinnaotong capsule on blood rheology of coronary heart disease patients combined with type 2 diabetes," China Practical Medical, vol. 7, pp. 154-155, 2012.

[6] L. Zhen, "Analysis the clinical application of Yindanxinnaotong capsule," Chinese Journal of Integrative Medicine on Cardio/Cerebrovascular Disease, vol. 11, pp. 248-250, 2013.

[7] W. H. W. Tang and Y. Huang, "Cardiotonic modulation in heart failure: insights from traditional Chinese medicine," Journal of the American College of Cardiology, vol. 62, no. 12, pp. 1073-1074, 2013.

[8] X. Li, J. Zhang, J. Huang et al., "A multicenter, randomized, double-blind, parallel-group, placebo-controlled study of the effects of qili qiangxin capsules in patients with chronic heart failure," Journal of the American College of Cardiology, vol. 62, no. 12, pp. 1065-1072, 2013.

[9] Y. Jia and S.-W. Leung, "Comparative efficacy of tongxinluo capsule and beta-blockers in treating angina pectoris: metaanalysis of randomized controlled trials," Journal of Alternative and Complementary Medicine, vol. 21, no. 11, pp. 686-699, 2015.

[10] C. Mao, X.-H. Fu, J.-Q. Yuan et al., “Tong-xin-luo capsule for patients with coronary heart disease after percutaneous coronary intervention," The Cochrane Database of Systematic Reviews, vol. 5, Article ID CD010237, 2015.

[11] H. Cao, J. Zhai, W. Mu et al., "Use of comparative effectiveness research for similar Chinese patent medicine for angina pectoris of coronary heart disease: a new approach based on patientimportant outcomes," Trials, vol. 15, no. 1, article 84, 2014.

[12] Y. Jia, F. Bao, F. Huang, and S.-W. Leung, "Is tongxinluo more effective than isosorbide dinitrate in treating angina pectoris? A systematic review and meta-analysis of randomized controlled trials," Journal of Alternative and Complementary Medicine, vol. 17, no. 12, pp. 1109-1117, 2011.

[13] H. Julian and G. Sally, Cochrane Handbook for Systematic Reviews of Interventions Version 5.1.0, 2011, http://www .cochrane.org/training/cochrane-handbook.

[14] A. R. Jadad, R. A. Moore, D. Carroll et al., "Assessing the quality of reports of randomized clinical trials: is blinding necessary?" Controlled Clinical Trials, vol. 17, no. 1, pp. 1-12, 1996.

[15] M. Tian, "Clinical study of Yindanxinnaotong capsule in the treatment of ischemic stroke," Medical Laboratory Science and Clinics, vol. 6, pp. 1548-1550, 2009.

[16] J.-Y. Zhang, "Analysis of curative effect of Yindanxinnaotong capsule in treatment of 65 cases of acute ischemic stroke," Chinese Journal of Modern Drug Application, pp. 124-125, 2014.

[17] J. Kang, "Yindanxinnaotong capsule combined with atorvastatin in the treatment of atherosclerotic cerebral infarction in 45 cases," Chinese Journal of Integrative Medicine on Cardio/Cerebrovascular Disease, vol. 9, pp. 948-949, 2011.

[18] T. Li and K.-T. Li, "Yindan Xinnaotong soft capsule in treating patients with cerebral infarction," Chinese Journal of Neuromedicine, vol. 10, pp. 1279-1281, 2011.

[19] L.-L. Ma, H. Liang, and J.-Y. Ren, "Clinical therapeutic effect of Yindanxinnaotong capsule on patients with lacunar infarction," Chinese Journal of Neuromedicine, vol. 10, no. 6, pp. 626-629, 2011.

[20] Y.-I. Shao, Y.-J. Runa, Z.-H. Gong, J.-X. He, D.-C. Xiang, and J. Qiu, "Clinical study on 'Yindan Xinnaotong Capsule' in Treating hypertension patient with residuals of cerebral infarction," Chinese Journal of Medical Guide, vol. 13, pp. 10151016, 2011.

[21] L.-M. Wu, "Effect of Yindanxinnaotong Capsule on hemorheology of cerebral infarction," Chinese Journal of Integrative Medicine on Cardio/Cerebrovascular Disease, vol. 10, pp. 11871188, 2012.

[22] S. Chen, "Observation of Yindanxinnaotong capsule treatment 50 cases of hypertension complicated with cerebral infarction," Journal of Practical Traditional Chinese Medicine, vol. 28, pp. 1050-1051, 2012.

[23] J. Yang, "Clinical observation of Yindanxinnaotong capsule on the treatment of acute ischemic stroke," Chinese Community Doctors, vol. 14, p. 231, 2012.

[24] B. Wang, "Yindanxinnaotong capsule in the treatment of ischemic stroke in 42 cases," Health Magazine, p. 169, 2012.

[25] H. Luo, "Clinical observation of Yindanxinnaotong capsule in the treatment of ischemic stroke," Health Magazine, vol. 11, no. 7, pp. 10-11, 2012.

[26] F.-W. Wang and Y.-L. Li, "Yindanxinnaotong capsule and cinepazide maleate in the treatment of vertebrobasilar ischemic vertigo," Chinese Journal of Integrative Medicine on Cardio/Cerebrovascular Disease, vol. 11, pp. 374-375, 2013.

[27] J. Yang, "Clinical observation on Yindan Xinnaotong capsule combined with aspirin in treatment of cerebral infarction sequelae," Research of Integrated Traditional Chinese and Western Medicine, no. 3, pp. 117-119, 2014.

[28] G.-Y. Lv and H.-J. Liu, "The therapeutic effect of Yindanxinnaotong capsule in the treatment of ischemic stroke," Hubei Journal of Traditional Chinese Medicine, vol. 21, pp. 2096-2098, 2012.

[29] F. Meng, "Analysis of therapeutic effect of Yindanxinnaotong capsule in the treatment of cerebral infarction," Contemporary Medicine, vol. 2, no. 6, p. 95, 2014.

[30] W.-W. Wang, Y.-R. Jin, L.-H. Zhao, and H. Cai, "Observation of Yindan Xinnaotong capsule in combination with aspirin on in plasma of patients with inflammatory factor of acute cerebral infarction and their secondary prevention effect," Chinese Journal of Integrative Medicine on Cardio/Cerebrovascular Disease, vol. 13, no. 11, pp. 1322-1323, 2015.

[31] Y. Su, Y. Wang, and X. Xiang, "Clinical observation on treatment of senile coronary heart disease and angina pectoris by Yindanxinnaotong," The Chinese Clinical Doctors, vol. 36, pp. 45-46, 2008.

[32] W. Yang, "The therapeutic effect of Yindanxinnaotong capsule combined with 3 kinds of chemical drugs in the treatment of angina pectoris of coronary heart disease," China Pharmacy, pp. 370-371, 2010.

[33] C.-Y. Yu, L.-L. Peng, and J.-Y. Yu, "Yindanxinnaotong capsules in treatment of chronic stable angina pectoris," Chinese Journal of Integrative Medicine on Cardio/Cerebrovascular Disease, vol. 8, pp. 1414-1415, 2010.

[34] H. Xiao, "Clinical observation on treatment of 36 cases of angina pectoris of Yindanxinnaotong," China's Health Care Nutrition, vol. 6, pp. 179-180, 2010.

[35] L. Li, "Clinical observation of Yindanxinnaotong capsule in treatment of angina pectoris of coronary heart disease," Chinese Journal of Integrative Medicine on Cardio/Cerebrovascular Disease, vol. 8, pp. 9-10, 2010.

[36] X. Zhao, "Aspirin and Yindanxinnaotong capsule in treatment of coronary heart disease in 30 cases of unstable angina 
pectoris," Chinese Journal of Integrative Medicine on Cardio/Cerebrovascular Disease, vol. 9, p. 1124, 2011.

[37] L.-Z. Hu and L. Xiao, "Effect of Yindanxinnaotong capsule on blood lipid in patients with unstable angina pectoris," Chinese Journal of Geriatric Heart Brain and Vessel Diseases, vol. 13, p. $561,2011$.

[38] X.-Y. Shang, Y. Li, J. Shi, and H.-Y. Hu, "Effects of YinDanXinNaoTong capsule on the levels of homocysteine and blood lipids in coronary heart disease patients," Chinese Journal of Geriatric Heart Brain and Vessel Diseases, vol. 13, pp. 122-124, 2011.

[39] F.-F. Luo, W.-J. Zhang, and X.-G. Li, "Observation of Yindanxinnaotong capsule in treatment of 50 cases of angina pectoris of coronary heart disease," Chinese Journal of Integrative Medicine on Cardio/Cerebrovascular Disease, vol. 9, pp. 66-367, 2011.

[40] G. He, "Yindanxinnaotong capsule in treatment of 30 cases of diabetes mellitus complicated with coronary heart disease and angina pectoris," Chinese Journal of Integrative Medicine on Cardio/Cerebrovascular Disease, vol. 9, pp. 879-880, 2011.

[41] Y. Guo, "Yindanxinnaotong capsule combined with isosorbide grease tablets in the treatment of stable angina pectoris and 32 cases of type," Chinese Journal of Integrative Medicine on Cardio/Cerebrovascular Disease, vol. 9, p. 999, 2011.

[42] X.-J. Jin and Q.-J. Song, "Clinical observation of Yindanxinnaotong capsule combined with Isosorbide Mononitrate Tablets on treatment of angina pectoris of coronary heart disease," Chinese and Foreign Medical Research, vol. 9, p. 40, 2011.

[43] S. Li, J. Li, and B.-Y. Zhou, "Efficacy observation of Yindanxinnaotong soft capsules combined with isosorbide 5-mononitrate and metoprolol tartrate tablet for coronary disease and angina pectoris," Chinese Medicine Modern Distance Education of China, vol. 9, pp. 59-60, 2011.

[44] L. Cai, "Clinical observation of Yindanxinnaotong capsule in the treatment of angina pectoris of coronary heart disease with diabetes mellitus," Hubei Journal of Traditional Chinese Medicine, vol. 33, pp. 37-38, 2011.

[45] H.-Y. Xing and F. Zhang, "The hemorheology of Yindanxinnaotong capsule in treatment of patients with coronary heart disease, angina pectoris and diabetes mellitus," Modern Journal of Integrated Traditional Chinese and Western Medicine, vol. 21, pp. 3806-3807, 2012.

[46] X. Shen, "Yindanxinnaotong capsule in treatment of coronary heart disease, diabetes and hyperlipidemia," Modern Journal of Integrated Traditional Chinese and Western Medicine, vol. 21, pp. 2080-2081, 2012.

[47] M.-Y. Shen and H.-X. Zhang, "The clinical efficacy and safety of Yindanxinnaotong capsule in the treatment of angina pectoris in senile coronary heart disease," Modern Journal of Integrated Traditional Chinese and Western Medicine, vol. 21, pp. 18721873, 2012.

[48] H.-S. Yuan, L.-M. Deng, D.-M. Wan, Q. Xu, and Q.-J. Zhang, "Yindanxinnaotong capsule in the treatment of qi stagnation and blood stasis of coronary heart disease and angina pectoris," Chinese Journal of Integrative Medicine on Cardio/Cerebrovascular Disease, vol. 10, pp. 1283-1284, 2012.

[49] B.-Y. Zhou, S. Li, and J. Li, "Clinical study of Yindanxinnaotong treatment of senile unstable angina pectoris," Modern Journal of Integrated Traditional Chinese and Western Medicine, vol. 21, pp. 3221-3222, 2012.

[50] K. Gu, "Yindanxinnaotong capsule in the treatment of type 2 diabetic patients with angina pectoris," Journal of Xinxiang Medical College, vol. 29, pp. 469-470, 2012.
[51] C. Gao, S.-Q. Chen, X.-Y. Bian, and C. Cui, "Combination treatment of traditional Chinese medicine in the treatment of coronary heart disease and angina pectoris combined with cervical spondylosis in 42 cases," Shanxi Journal of Traditional Chinese Medicine, vol. 24, pp. 27-28, 2012.

[52] Q. Li, "The clinical therapeutic effect of Yindanxinnaotong capsule in treatment of angina pectoris of coronary heart disease," Chinese and Foreign Medical Research, vol. 8, pp. 3738, 2013.

[53] L.-J. Zhou, X. Zhang, J. Lv, and R.-H. Zhou, "Yindanxinnaotong capsule in the treatment of phlegm type coronary heart disease angina pectoris," Chinese Journal of Integrative Medicine on Cardio/Cerebrovascular Disease, vol. 11, pp. 17-18, 2013.

[54] Z.-L. Qiao, J.-F. Guo, and L.-L. Qi, "Clinical observation of 90 cases of Yindanxinnaotong capsule in treatment of angina pectoris," Chinese Journal of Integrative Medicine on Cardio/Cerebrovascular Disease, vol. 11, pp. 664-665, 2013.

[55] L. Xu, S.-M. Deng, and G. Sun, "The therapeutic effect of Yindanxinnaotong capsule in the treatment of qi stagnation and blood stasis in patients with stable angina pectoris," Chinese Journal of Integrative Medicine on Cardio/Cerebrovascular Disease, p. 1149, 2014.

[56] N. Yan, X. Zhang, and F. Li, “The treatment effect of Yindanxinnaotong capsule in 50 cases of coronary heart disease angina pectoris," Medical Information, vol. 14, pp. 33-35, 2014.

[57] X. Du, "Effect of Yindanxinnaotong Capsule on plasma homocysteine (Hcy) and blood lipid levels in patients with qi stagnation and blood stasis type," Nei Mongol Journal of Traditional Chinese Medicine, vol. 33, pp. 1-2, 2014.

[58] X.-Y. Du, "Effect of Yindanxinnaotong Capsule on Hcy and blood lipid levels in patients with qi stagnation and blood stasis type in patients with coronary heart disease," Nei Mongol Journal of Traditional Chinese Medicine, vol. 33, pp. 1-2, 2014.

[59] P. Zhang, "Clinical observation of 60 cases of Yindanxinnaotong capsule in treatment of angina pectoris of coronary heart disease," For All Health, vol. 3, p. 18, 2014.

[60] G.-B. Lan, Z.-Q. Wang, and S.-X. Cai, "The therapeutic effect of Yindanxinnaotong capsule in the treatment of senile coronary heart disease," Chinese Journal of Integrative Medicine on Cardio/Cerebrovascular Disease, pp. 671-672, 2014.

[61] G.-F. Tuo and H.-H. Shen, "Observe the clinical effect of Yindan Xinnaotong capsule in treatment of angina pectoris," Nei Mongol Journal of Traditional Chinese Medicine, vol. 33, pp. 29-30, 2014.

[62] Y. Huang, J. Guo, and H. Fan, "Clinical observation of Yindanxinnaotong capsule in treatment of angina pectoris of coronary heart disease," Chinese Journal of Integrative Medicine on Cardio/Cerebrovascular Disease, vol. 5, pp. 619-620, 2014.

[63] J. Liu, Z. Chen, W. Zhang, and J. Qin, “The clinical effect of Yindanxinnaotong on patients with coronary heart disease and angina pectoris," Chinese Journal of Experimental Traditional Medical Formulae, vol. 13, pp. 61-62, 2007.

[64] J. C. de la Torre, "Cerebrovascular and cardiovascular pathology in Alzheimer's disease," International Review of Neurobiology, vol. 84, pp. 35-48, 2009.

[65] P. Giannoni, M. Arango-Lievano, I. D. Neves et al., "Cerebrovascular pathology during the progression of experimental Alzheimer's disease," Neurobiology of Disease, vol. 88, pp. 107117, 2016. 
[66] M. Riverol, J. T. Becker, O. L. López et al., "Relationship between systemic and cerebral vascular disease and brain structure integrity in normal elderly individuals," Journal of Alzheimer's Disease, vol. 44, no. 1, pp. 319-328, 2015.

[67] Y. Liu, M. Guo, L. Xu, C.-G. Fu, and K.-J. Chen, "Cardiovascular pharmacological effects of Ginkgo preparations in Chinese medicine: mechanism and prospect," in Proceedings of the 16th International Symposium on Cardiovascular Diseases in South China, vol. 6, pp. 543-550, 2014.

[68] J. Wang, L. Wang, H. Yang et al., "Prevention of atherosclerosis by Yindan Xinnaotong capsule combined with swimming in rats," BMC Complementary and Alternative Medicine, vol. 15, no. 1, article 109, 2015.

[69] W. Wang, L. Wang, H. Yang et al., "Protective effects of Yindanxinnaotong capsule in a rat model of myocardial ischemia/reperfusion injury," Journal of Traditional Chinese Medicine, vol. 34, no. 6, pp. 699-709, 2014.

[70] L. Gong, H. Xu, L. Wang et al., "Identification and evaluation of the chemical similarity of Yindan xinnaotong samples by ultra high performance liquid chromatography with quadrupole time-of-flight mass spectrometry fingerprinting," Journal of Separation Science, vol. 39, pp. 611-622, 2016.

[71] X.-L. Piao and Q. Wu, "Progressive studies on gynostemma pentaphyllum," Lishizhen Medicine and Materia Medica Research, vol. 21, pp. 1758-1760, 2010. 


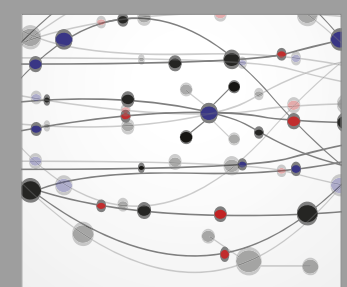

The Scientific World Journal
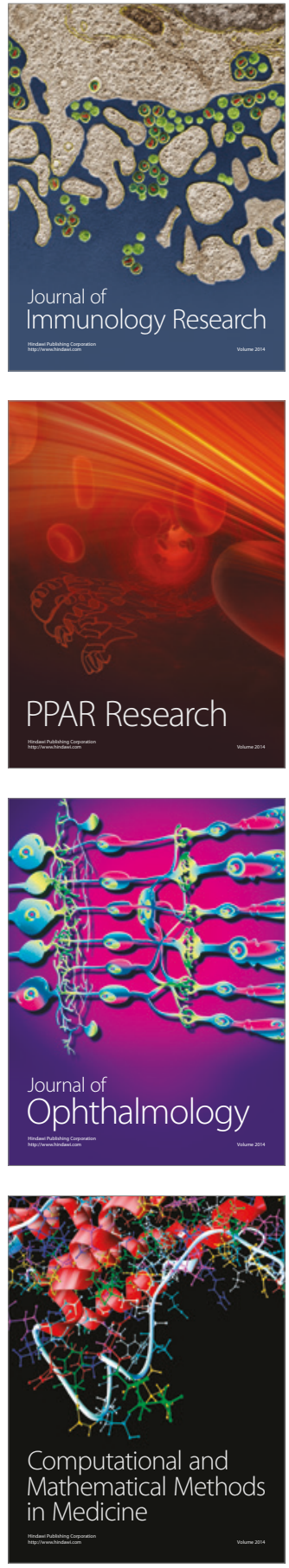

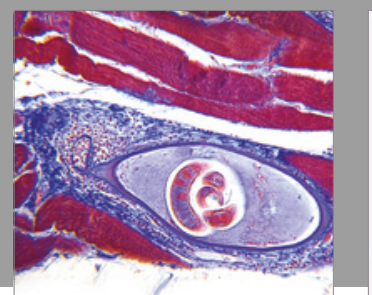

Gastroenterology Research and Practice
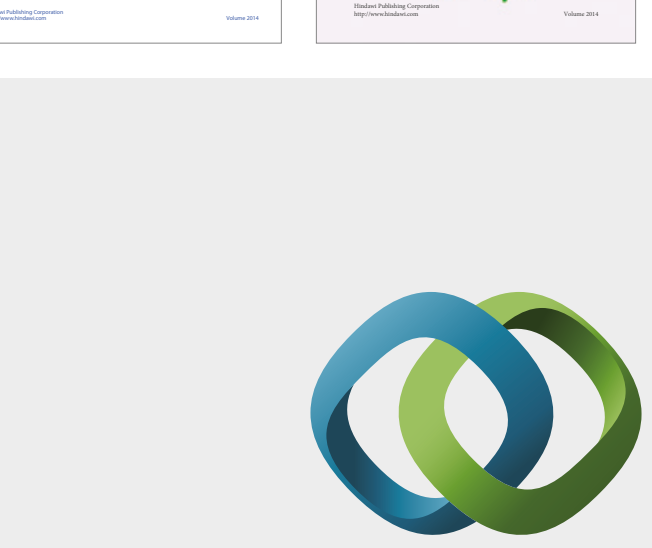

\section{Hindawi}

Submit your manuscripts at

https://www.hindawi.com
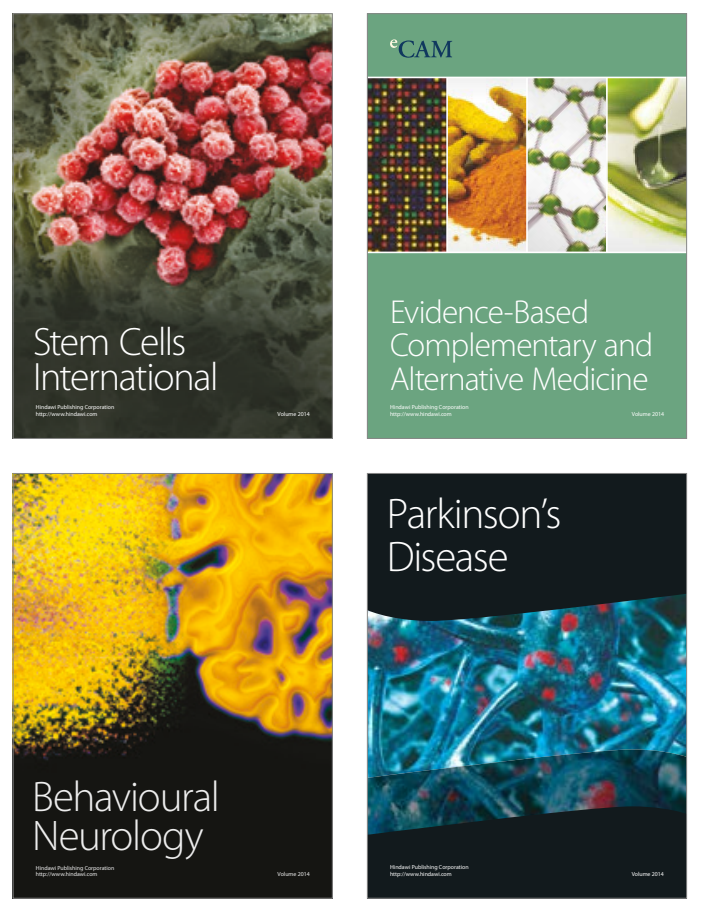
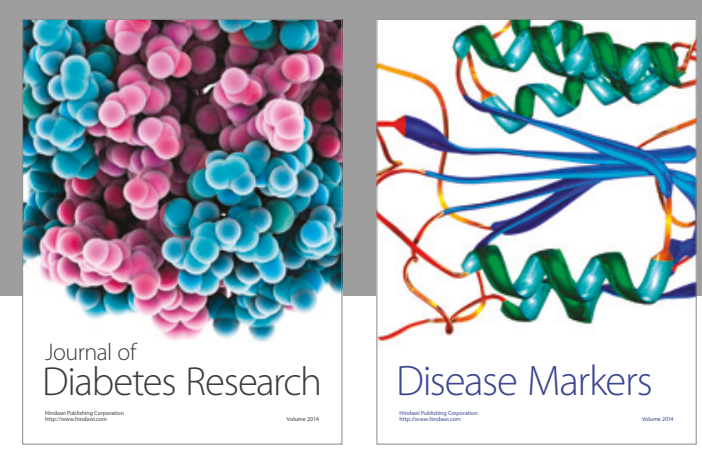

Disease Markers
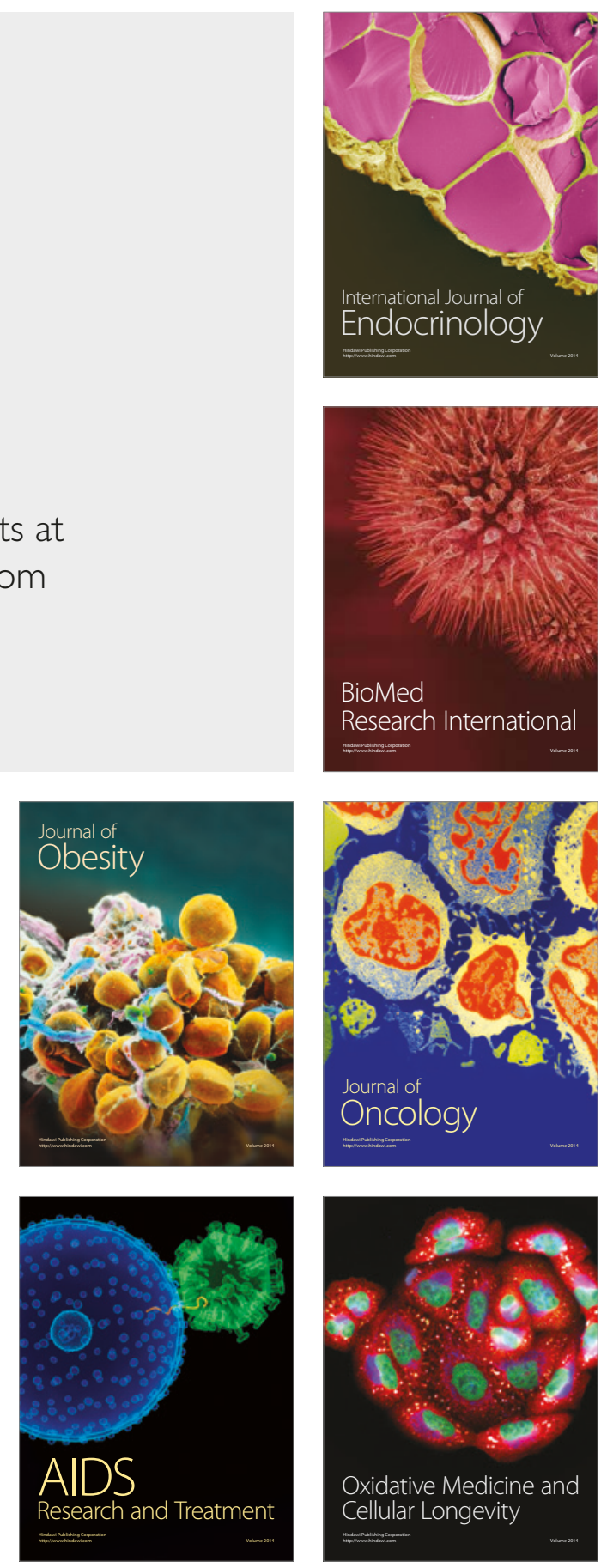